\title{
EGR1 Is a Critical Gene in Response of Human Keratinocyte to Blue Light Radiation
}

\author{
Maryam Hamzeloo-Moghadam ${ }^{\mathbb{D}}$, Mostafa Rezaei Tavirani ${ }^{*}{ }^{\circledR}$, Mohhamadreza $\operatorname{Razzaghi}^{\left.{ }^{(}\right)}$, Sina

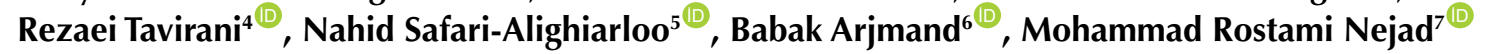 \\ ${ }^{1}$ Traditional Medicine and Material Medical Research Center, School of Traditional Medicine, Shahid Beheshti \\ University of Medical Sciences, Tehran, Iran \\ ${ }^{2}$ Proteomics Research Center, Faculty of Paramedical Sciences, Shahid Beheshti University of Medical Sciences, \\ Tehran, Iran \\ ${ }^{3}$ Laser Application in Medical Sciences Research Center, Shahid Beheshti University of Medical Sciences, Tehran, Iran \\ ${ }^{4}$ Proteomics Research Center, Shahid Beheshti University of Medical Sciences, Tehran, Iran \\ ${ }^{5}$ Endocrine Research Center, Institute of Endocrinology and Metabolism, Iran University of Medical Sciences, \\ Tehran, Iran \\ ${ }^{6}$ Cell Therapy and Regenerative Medicine Research Center, Endocrinology and Metabolism Molecular-Cellular \\ Sciences Institute, Tehran University of Medical Sciences, Tehran, Iran \\ ${ }^{7}$ Research Institute for Gastroenterology and Liver Diseases, Gastroenterology and Liver Diseases Research Center, \\ Shahid Beheshti University of Medical Sciences, Tehran, Iran
}

\section{*Correspondence to Mostafa Rezaei Tavirani, Email: tavirany@yahoo.com}

Received: June 11, 2021 Accepted: October 22, 2021 Published online 26 December 2021

\section{Introduction}

Several types of lasers are applied in different fields of medicine and also for cosmetic purposes. ${ }^{1,2}$ Since the benefits of laser application are significant, it can be expected that the use of lasers will achieve considerable progress in the near future. ${ }^{3}$ On the other hand, the molecular mechanism of laser effects on the human body is not clear yet. ${ }^{4}$ There are many documents about the molecular mechanism regarding the gene expression change of many genes after the treatment of the human body by diverse lasers. ${ }^{5}$ In vitro and also in vivo investigations represent useful results, along with contradictory data about the effect of low-level lasers in medicine. $^{5}$

Gene expression studies have attempted to elucidate the molecular mechanism of many diseases and administrate interventions. ${ }^{6}$ In such studies, gene expression changes in the treated samples were compared with the controls to find pure effects of the applied condition or probes. ${ }^{7}$ Since a large number of genes usually dysregulate, it is necessary to find the critical dysregulated gene or genes. Network analysis is a useful method to screen the dysregulated genes based on the role of the studied genes in interaction with the other elements of the network. ${ }^{8}$

A network contains nodes which are connected together via edges. ${ }^{9}$ In the scale-free network, there are a few nodes that play an important role in constructing the network. These nodes are known as central nodes. ${ }^{10}$ One type of important central node is recognized as hubs. The hub nodes are characterized by a large number of connections (adages) which the hub node makes with the other nodes directly (this centrality parameter is identified as

Please cite this article as follows: Hamzeloo-Moghadam M, Rezaei Tavirani M, Razzaghi M, Rezaei Tavirani S, Safari-Alighiarloo N, Arjmand B, et al. EGR1 is a critical gene in response of human keratinocyte to blue light radiation. J Lasers Med Sci. 2021;12:e83. doi:10.34172/jlms.2021.83. 
degree) ${ }^{11}$ Betweenness is the other centrality parameter that refers to the related shortest paths of a node. A node that is characterized by a high value of betweenness is known as bottleneck. A node that is hub and bottleneck is determined as hub-bottleneck which is a strong central node. $^{12,13}$ There are other centrality parameters such as closeness centrality and stress that refer to the more effective role of the studied node in the structure of the constructed network. ${ }^{14}$ A node characterized by a greater number of high-value centrality parameters plays a critical role in the analyzed network. ${ }^{15}$

In the present study, the gene expression profiles of human keratinocytes which were treated by one-hour blue light radiation were compared with the controls and the significant differentially expressed genes (DEGs) were determined to construct a network. The critical elements of the network were determined and discussed to find a prominent effect of blue light on the human body.

\section{Methods}

Data of GSE89086 (which is entitled "Photobiomodulation bybluelightinducesadosedependentbiphasicproliferation curve in human keratinocytes") were downloaded from Gene Expression Omnibus (GEO). (https://www.ncbi. nlm.nih.gov/geo/query/acc.cgi?acc=GSE89086). As it is reported in this document, three treated samples of human keratinocytes were exposed to 7.5 minutes blue light irradiation and then were harvested $1 \mathrm{~h}$ after irradiation. These samples were characterized as GSM2358577, GSM2358579, and GSM235881. As the treated individuals without light irradiation, the three control samples (including GSM2358578, GSM2358580, and GSM235882) were similar. The gene expression profiles of the treated samples versus the control cells were analyzed via GEO2R.

A volcano plot, a Meandiff plot, and an expression density curve were provided to compare the treated and control gene expression profiles. Considering $P$ value $<0.05$ and fold change $>1.5$, the significant DEGs were extracted. The name and description of the significant DEGs were searched via GEO "Accession viewer-NCBI" (https://www.ncbi.nlm.nih.gov/geo/query/acc.cgi). The names of the selected DEGs were searched in the STRING database ${ }^{16}$ and the recognized ones plus 100 first neighbors from the STRING database were interacted by Cytoscape software. ${ }^{17}$ The network was analyzed by "networkAnalyzer" application of Cytoscape. To find the central nodes, $10 \%$ of the top nodes based on degree value, betweenness centrality, closeness centrality, and stress were identified. The common nodes among the central nodes were determined as critical nodes. Based on the amounts of the centrality parameters, the crucial nodes were selected among the critical nodes. The relationship between the crucial nodes and their first neighbors was investigated via CluePedia application of Cytoscape software.

\section{Results}

Gene expression profile analysis by GEO2R revealed that the profiles of treated and control cells are comparable. As it is shown in Figure 1, only a few numbers of genes are dysregulated statistically and most dysregulated genes do not have significant expression changes. In this figure, asymmetric distribution between up- and down-regulated genes is presented. The distribution of the dysregulated genes based on the expression value versus the logarithm of fold change is shown in Figure 2. As it is presented in this figure, an increase of fold change or gene express change is accompanied by a decrease in the numbers of genes without considering the up- or down-regulation modes of expression change. The density of expression for the dysregulated genes is shown in Figure 3. Based on the presented curve in Figure 3, the density of expression is maximized in the low value of the intensity of expression. It can be considered that the contribution of highly dysregulated genes to the density of expression versus the low or mild individuals is characterized by lower or

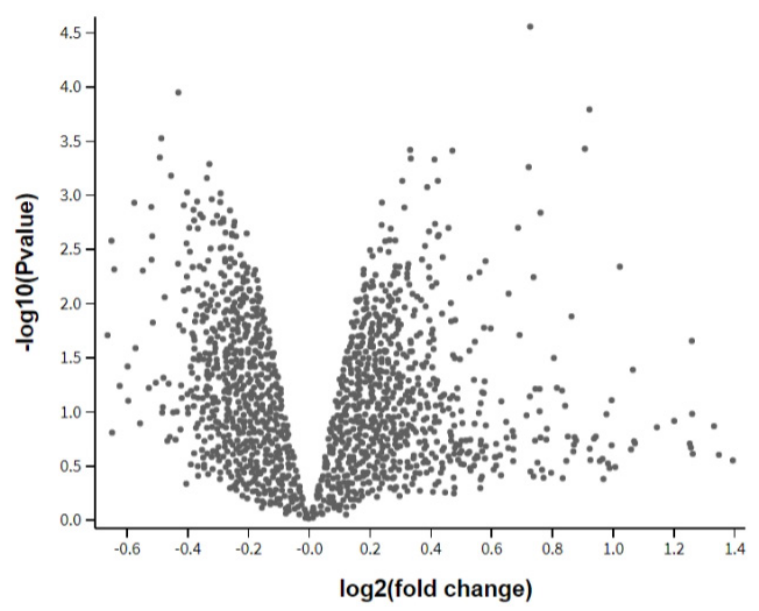

Figure 1. The Volcano Plot of the Gene Expression Profile of Human Keratinocytes in the Presence of 7.5 min Blue Light Radiation Versus the Controls.

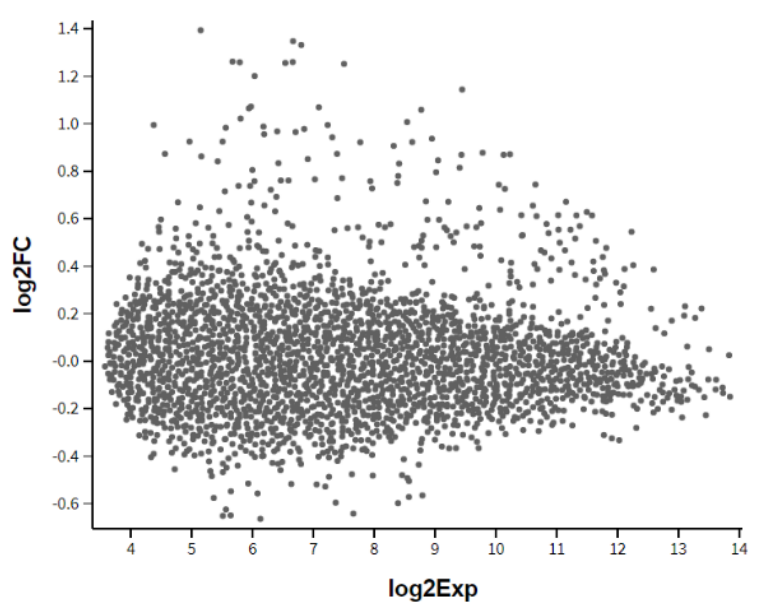

Figure 2. The Meandiff Plot of the Gene Expression Profile of Human Keratinocytes in the Presence of 7.5 min Blue Light Radiation Versus the Controls. The logarithm of expression change values versus the logarithm of fold changes is presented for the related dysregulated genes. 
minimum quantities.

The analysis led to the introduction of 6 characterized significant DEGs. The names and descriptions of these

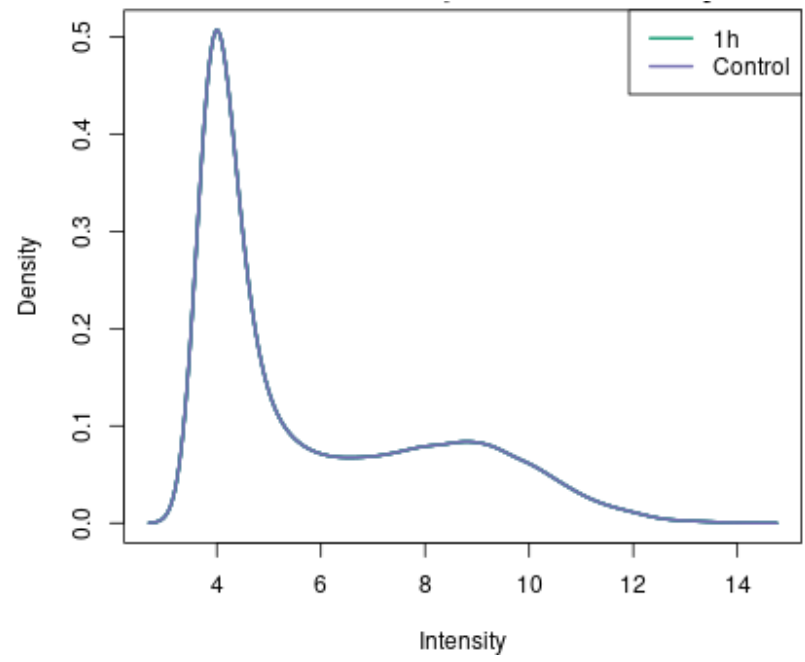

Figure 3. Presentation of the Density of Expression Versus the Intensity of Expression for Gene Expression Profile of Human Keratinocytes in the Presence of 7.5 min Blue Light Radiation Versus the Controls. genes are tabulated in Table 1. Four characterized significant DEGs including EID3, ARRDC3, PLEKHM1, and EGR1 were recognized by the STRING database. The network was constructed for the 4 recognized DEGs plus 100 first neighbors (see Figure 4). The 104 elements of the network were connected by 1530 undirected connections. The network was analyzed by "NetworkAnalyzer" and visualized based on degree value. Among the 40 central nodes 3 critical individuals including EGR1, STAT1, and IGS15, which are presented in Table 2, were identified. Since EGR1 was eligible to be selected as a crucial node, 10 first neighbors of this gene were extracted from the STRING database. The action map of EGR1 and its 10 first neighbors are shown in Figure 5.

\section{Discussion}

There are many documents about gene expression changes and photobiomodulation (PBM). In such references, researchers have shown that after light radiation several genes are dysregulated. ${ }^{18,19}$ Since the understanding of the molecular mechanism of PBM effects on the body and human cells is an important feature of the therapeutic

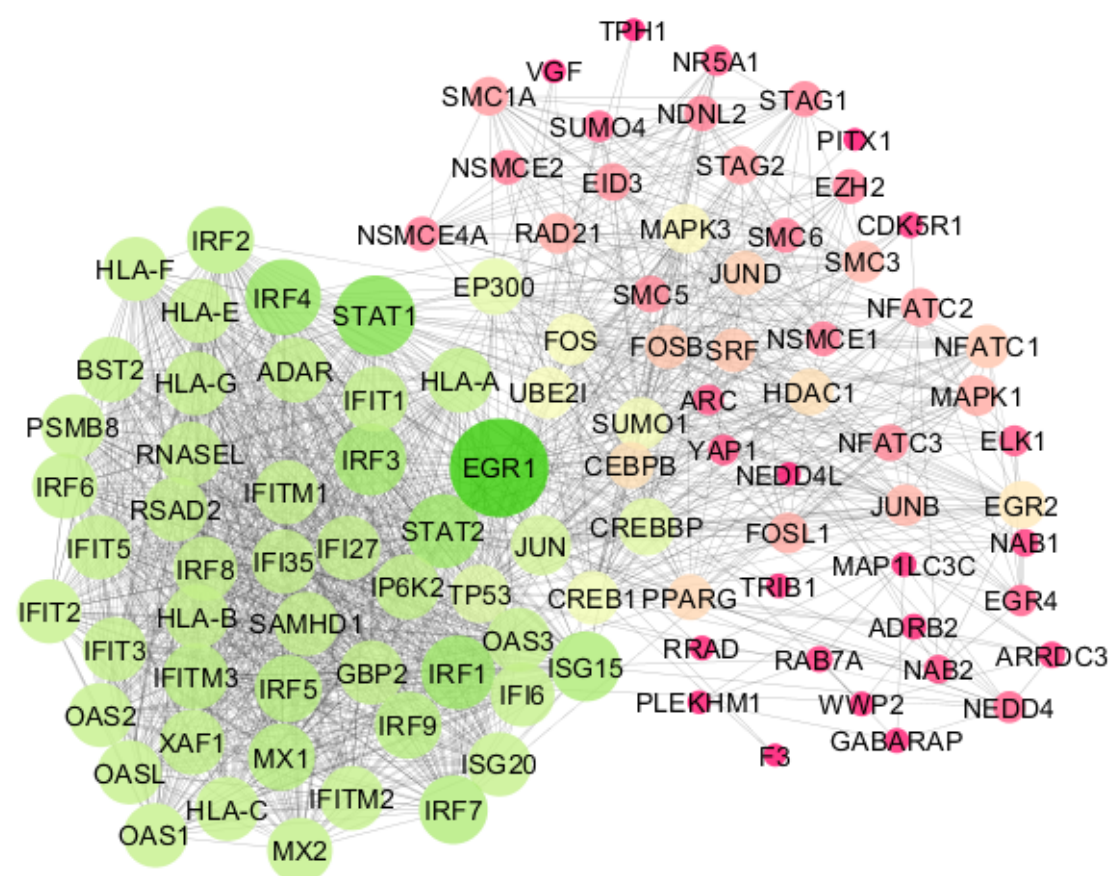

Figure 4. A Network Including 4 Queried DEGs and the 100 First Neighbors Which Differentiate Human Keratinocytes in the Presence of 7.5 min Blue Light Radiation From the Control Individuals. The nodes are layout based on degree value; the bigger size and green color refer to a higher value of degree.

Table 1. The Characterized Significant DEGs Which Differentiate Human Keratinocytes in the Presence of 7.5 min Blue Light Radiation From the Controls.

\begin{tabular}{|c|c|c|c|c|c|}
\hline No. & Gene ID & Gene description & Display name & $P$ Value & $\operatorname{LogFC}$ \\
\hline 1 & 344887_at & NmrA-like family domain containing 1 pseudogene & NMRAL2P & 0.0002 & 0.922 \\
\hline 2 & 493861_at & EP300 interacting inhibitor of differentiation 3 & EID3 & 0.0004 & 0.907 \\
\hline 3 & 28809_at & Immunoglobulin lambda variable 3-1 & IGLV3-1 & 0.0014 & 0.761 \\
\hline 4 & 9842_at & Pleckstrin homology domain containing family M (with RUN domain) member 1 & PLEKHM1 & 0.0000 & 0.727 \\
\hline 5 & 1958_at & Early growth response 1 & EGR1 & 0.0020 & 0.687 \\
\hline 6 & 57561_at & Arrestin domain containing 3 & ARRDC3 & 0.0048 & -0.642 \\
\hline
\end{tabular}


Table 2. Critical Nodes of the Analyzed Network

\begin{tabular}{cccccc}
\hline No. & Gene Display Name & Degree & BC & CC & Stress \\
\hline 1 & EGR1 & $80^{1}$ & $0.196^{1}$ & $0.792^{1}$ & $8738^{1}$ \\
2 & STAT1 & $61^{2}$ & $0.044^{5}$ & $0.696^{2}$ & $5318^{5}$ \\
3 & ISG15 & $50^{7}$ & $0.027^{8}$ & $0.656^{3}$ & $3482^{8}$ \\
\hline
\end{tabular}

Note: BC and CC refer to betweenness centrality and closeness centrality respectively. Superscript number corresponds to the rank of the node among the central individuals based on the titled centrality parameters.

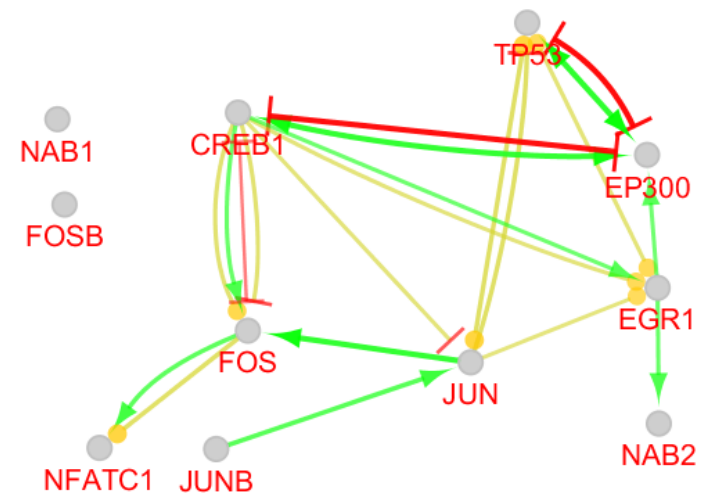

Figure 5. The Action Map of EGR1 and its 10 First Neighbors. Green, red, and yellow colors refer to activation, inhibition, and expression actions respectively.

properties of PBM, great efforts have been made to find the key targets of the exposure. On the other hand, network analysis is a well-established method to study dysregulated genes. ${ }^{20}$

As it is shown in Figure 1, most genes of the human keratinocytes are not affected by blue light significantly. Therefore, it can be concluded that a limited number of dysregulated genes can be identified. Dispersion of genes on the right side of Figure 1 indicates that up-regulation is a dominant process versus downregulation in the cells which were exposed to blue light. This finding corresponds with other researches that refer to more upregulated DEGs relative to less downregulated ones. ${ }^{21}$ The Meandiff plot (see Figure 2) shows gross changes of upregulated DEGs versus the downregulate genes obviously. As it is depicted in Figure 3, the expression of most genes is not affected noticeably by exposure. On the other hand, $7.5 \mathrm{~min}$ blue light radiation is accompanied by mild alteration in the gene expression process of human keratinocytes. Mild effects of PBM are reported by several researchers that applied PBM as a therapeutic tool. ${ }^{22}$

As it is depicted in Table 1, there are 6 characterized DEGs that differentiate the treated samples from the control cells. However, only four DEGs were recognized by the database. Network analysis revealed that EGR1 is the crucial dysregulated gene which is targeted by blue light radiation. Based on the results of this analysis, EGR1 occupies the first rank among the central nodes (including the queried DEGs and the added first neighbor genes) regarding degree value, betweenness centrality, closeness centrality, and stress as the four important centrality parameters (see Table 2). Degree and betweenness of EGR1 are $33 \%$ and $168 \%$, which are higher than STAT1 and UBE2I as the second hub and second bottleneck genes respectively. It can be concluded that EGR1 is the key gene that is targeted by blue light radiation. As it is represented in Table 1, EGR1 is upregulated after 1-hour radiation.

It is reported that EGR1 belongs to the family of immediate early response transcription factors which plays a role in several important cellular processes such as stress responses, cell development, cell growth, and regulating of homeostasis of stem cells in many tissues. ${ }^{23}$ Several key roles such as the gatekeeper of inflammatory enhancers of human macrophages, the multifaceted regulator of matrix production in tendons and other connective tissues, the regulator of cell cycle progression and tumorigenesis in gastric cancer are attributed to EGR1. ${ }^{24-26}$

As it is depicted in Figure 5, CREB1, EP300, FOS, FOSB, JUN, JUNB, NAB1, NAB2, NFATC1, and TP53 are the first neighbors of EGR1. CREB1, EP300, JUN, NAB2, and TP53 are directly connected to EGR1. Since TP53, EP300, and JUN are central nodes, the relationship between EGR1 and these 3 first neighbors could be important. As it is shown in Figure 5, EGR1 activates EP300 but upregulates TP53. The single connection between JUN and EGR1 refers to the upregulation of EGR1 by JUN. Many years ago, JUN was characterized as a proto-oncogene. ${ }^{27}$ The upregulation of EGR1 by JUN refers to the anticancer property of EGR1 which increases against tumor development. There are many documents about cancer onset and autism due to mutation and partial deletion of EP300. ${ }^{28,29}$ It seems that the activation of EP300 by EGR1 is a protective role of EGR1 against the mentioned diseases. TP53 is a well-known tumor suppressor gene. ${ }^{30}$ The upregulation of TP53 by upregulated EGR1 due to blue light radiation indicates the cancer prevention effect of PBM.

\section{Conclusion}

It can be concluded that 1-hour bluelight radiation induced the gene expression change of many genes of human keratinocytes; however, only a few genes were affected significantly. The results indicate that upregulation is a prominent process. ERG1 as a key upregulated gene was highlighted in the network analysis. The finding indicates that EGR1 plays a protecting role against cancer and stress in the irradiated cells. More investigations by using different cell types are suggested.

\section{Ethical Considerations}

Not applicable.

\section{Conflict of Interests}

The authors declare they have no conflicts of interest. 


\section{Acknowledgment}

Shahid Beheshti University of Medical Sciences supported this research.

\section{References}

1. Vitale M, Truchuelo T. 13270 Evaluation of the effects of a cosmetic treatment based on the secretion of Cryptomphalus aspersa $40 \%$ after the use of ablative fractionated $\mathrm{CO} 2$ laser in cutaneous aging. J Cosmet Dermatol. 2020;19(3):622-628. doi: 10.1111/jocd.13052.

2. Hu M-L, Zheng G, Lin H, Li N, Zhao P-F, Han J-M. Network meta-analysis of the treatment efficacy of different lasers for peri-implantitis. Lasers Med Sci. 2021;36(3):619-629. doi: 10.1007/s10103-020-03101-3.

3. Mirza FN, Mirza HN, Khatri KA. Concomitant use of isotretinoin and lasers with implications for future guidelines: An updated systematic review. Dermatol Ther. 2020;33(6):e14022. doi: 10.1111/dth.14022.

4. Shimohira T, Katagiri S, Ohsugi Y, Hirota T, Hatasa M, Mizutani K, et al. Comprehensive and sequential gene expression analysis of bone healing process following Er: YAG laser ablation. Photobiomodul Photomed Laser Surg. 2021;39(2):100-112. doi: 10.1089/photob.2020.4833.

5. Amaroli A, Colombo E, Zekiy A, AicardiS, BenedicentiS, De Angelis N. Interaction between laser light and osteoblasts: photobiomodulation as a trend in the management of socket bone preservation-a review. Biology (Basel). 2020;9(11):409. doi: 10.3390/biology9110409.

6. Kang GG, Francis N, Hill R, Waters D, Blanchard C, Santhakumar AB. Dietary polyphenols and gene expression in molecular pathways associated with type 2 diabetes mellitus: A Review. Int J Mol Sci. 2019 ;21(1):140. doi: 10.3390/ijms21010140.

7. Jang K, Tong T, Lee J, Park T, Lee H. Altered Gene Expression Profiles in Peripheral Blood Mononuclear Cells in Obese Subjects. Obes Facts. 2020;13:375-385. doi: 10.1159/000507817.

8. Cordero AIH, Li X, Yang CX, Milne S, Bossé Y, Joubert P, et al. Gene expression network analysis provides potential targets against SARS-CoV-2. Sci Rep. 2020;10(1):1-9. doi: 10.1038/s41598-020-78818-w

9. Peyvandi H, Peyvandi AA, Safaei A, Azodi MZ, RezaeiTavirani M. Introducing potential key proteins and pathways in human laryngeal cancer: a system biology approach. Iran J Phrama Res. 2018;17(1):415-25. doi: 10.22037/IJPR.2018.2173

10. Zamanian-Azodi M, Arjmand B, Razzaghi M, Rezaei Tavirani M, Ahmadzadeh A, Rostaminejad M. Platelet and Haemostasis are the Main Targets in Severe Cases of COVID-19 Infection; a System Biology Study. Arch Acad Emerg Med. 2021; 9(1): e27. doi: 10.22037/aaem.v9i1.1108.

11. Zamanian-Azodi M, Hajisayah SK, Razzaghi M, RezaeiTavirani M. Introducing physical exercise as a potential strategy in liver cancer prevention and development. Gastroenterol Hepatol Bed Bench.2021;14(4):317-322

12. Rostami-Nejad M, Rezaei-Tavirani M, Zadeh-Esmaeel M-M, RezaeiTavirani S, Akbari Z, Esmaeili S, et al. Assessment of cytokine-mediated signaling pathway dysregulation in arm skin after $\mathrm{CO} 2$ laser therapy. J Lasers Med Sci. 2019;10(4):257-263. doi: 10.15171/jlms.2019.42.
13. Hazra S, Chaudhuri AG, Tiwary BK, Chakrabarti N. Matrix metallopeptidase 9 as a host protein target of chloroquine and melatonin for immunoregulation in COVID-19: A network-based meta-analysis. Life Sci. 2020;257:118096. doi: 10.1016/j.lfs.2020.118096.

14. Manian V, Gangapuram H, Orozco J, Janwa H, Agrinsoni C. Network Analysis of Local Gene Regulators in Arabidopsis thaliana under Spaceflight Stress. Computers. 2021;10(2):18. doi: 10.3390/computers 10020018

15. Soofi A, Taghizadeh M, Tabatabaei SM, Tavirani MR, Shakib $\mathrm{H}$, Namaki S, et al. Centrality analysis of protein-protein interaction networks and molecular docking prioritize potential drug-targets in type 1 diabetes. Iran J Phrama Res. 2020;19(4):121-134. doi: 10.22037/ijpr.2020.113342.14242

16. Szklarczyk D, Gable AL, Nastou KC, Lyon D, Kirsch R, Pyysalo S, et al. The STRING database in 2021: customizable protein-protein networks, and functional characterization of user-uploaded gene/measurement sets. Nucleic Acids Res. 2021;49(D1):D605-D12. doi: 10.1093/nar/gkaa1074

17. Otasek D, Morris JH, Bouças J, Pico AR, Demchak B. Cytoscape automation: empowering workflow-based network analysis. Genome Biol. 2019;20(1):1-15. doi: 10.1186/s13059-019-1758-4

18. Ayuk SM, Abrahamse H, Houreld NN. The role of photobiomodulation on gene expression of cell adhesion molecules in diabetic wounded fibroblasts in vitro. Journal of Photochemistry and Photobiology B: J Photochem Photobiol B. 2016;161:368-74. doi: 10.1016/j. jphotobiol.2016.05.027.

19. Ruh AC, Frigo L, Cavalcanti MFXB, Svidnicki P, Vicari VN, Lopes-Martins RAB, et al. Laser photobiomodulation in pressure ulcer healing of human diabetic patients: gene expression analysis of inflammatory biochemical markers. Lasers Med Sci. 2018;33(1):165-171. doi: 10.1007/s10103017-2384-6.

20. Jia-Juan Tu, Le Ou-Yang, Yuan Zhu, Hong Yan, Hong Qin, Xiao-Fei Zhang, Differential network analysis by simultaneously considering changes in gene interactions and gene expression. Bioinformatics. 2021;btab502. doi:10.1093/bioinformatics/btab502

21. Antunes HS, Wajnberg G, Pinho MB, Jorge NAN, de Moraes JLM, Stefanoff CG, et al. cDNA microarray analysis of human keratinocytes cells of patients submitted to chemoradiotherapy and oral photobiomodulation therapy: pilot study. Lasers Med Sci. 2018;33(1):11-18. doi: 10.1007/ s10103-017-2313-8.

22. Locke RC, Lemmon EA, Dudzinski E, Kopa SC, Wayne JM, Soulas JM, et al. Photobiomodulation does not influence maturation and mildly improves functional healing of mouse achilles tendons. J Orthop Res. 2020;38(8):18661875. doi: 10.1002/jor.24592.

23. Min IM, Pietramaggiori G, Kim FS, Passegué E, Stevenson KE, Wagers AJ. The transcription factor EGR1 controls both the proliferation and localization of hematopoietic stem cells. Cell Stem Cell. 2008;2(4):380-91. doi: 10.1016/j. stem.2008.01.015.

24. Trizzino M, Zucco A, Deliard S, Wang F, Barbieri E, Veglia F, et al. EGR1 is a gatekeeper of inflammatory enhancers in human macrophages. Sci Adv. 2021;7(3):eaaz8836. doi: 10.1126/sciadv.aaz8836. 
25. Havis E, Duprez D. EGR1 transcription factor is a multifaceted regulator of matrix production in tendons and other connective tissues. Int J Mol Sci. 2020;21(5):1664. doi: 10.3390/ijms21051664.

26. Ma Z, Gao X, Shuai Y, Wu X, Yan Y, Xing X, et al. EGR1mediated linc01503 promotes cell cycle progression and tumorigenesis in gastric cancer. Cell Prolif. 2021;54(1):e12922. doi: 10.1111/cpr.12922.

27. Angel P, Hattori K, Smeal T, Karin M. The jun protooncogene is positively autoregulated by its product, Jun/AP-1. Cell. 1988;55(5):875-85. doi: 10.1016/00928674(88)90143-2.

28. Zhu D, Ren X, Luo Y, Huang B, Huang J. Bladder Malignant Granular Cell Tumor with EP300 Gene Mutation: A
Case Report and Literature Review. J Cancer Ther. 2021;12(5):240-53. doi: 10.4236/jct.2021.125023

29. Brewer CJ, Padeganeh A, Ghochani M, Hajianpour A, Moradian MM, Ramjit RT. 8. A partial deletion of the EP300 gene detected by microarray in an individual with mild autism. Cancer Genet. 2021;252(suppl 1):S3. doi: 10.1016/j.cancergen.2021.01.019

30. Velez MG, Kosiorek HE, Egan JB, McNatty AL, Riaz IB, Hwang SR, et al. Differential impact of tumor suppressor gene (TP53, PTEN, RB1) alterations and treatment outcomes in metastatic, hormone-sensitive prostate cancer. Prostate Cancer Prostatic Dis. 2021:1-5. doi: 10.1038/ s41391-021-00430-4 